Article

\title{
Preparation and Characterization for Antibacterial Activities of 3D Printing Polyetheretherketone Disks Coated with Various Ratios of Ampicillin and Vancomycin Salts
}

\author{
Ngi-Chiong Lau ${ }^{1,2}$, Min-Hua Tsai ${ }^{1,3}$, Dave W. Chen ${ }^{1,2}$, Chien-Hao Chen ${ }^{1,2}$ and \\ Kong-Wei Cheng ${ }^{1,3, *}$ \\ 1 Department of Orthopaedic Surgery, Chang Gung Memorial Hospital, Keelung Branch, Keelung 204, \\ Taiwan; edbui11@hotmail.com (N.-C.L.); m0523048@stmail.cgu.edu.tw (M.-H.T.); \\ mr5181@adm.cgmh.org.tw (D.W.C.); chchen1982@gmail.com (C.-H.C.) \\ 2 College of Medicine, Chang Gung University, Taoyuan 333, Taiwan \\ 3 Department of Chemical and Materials Engineering, Chang Gung University, Taoyuan 333, Taiwan \\ * Correspondence: kwcheng@mail.cgu.edu.tw
}

Received: 30 October 2019; Accepted: 16 December 2019; Published: 20 December 2019

Featured Application: Bone Engineering, Antibacterial materials.

\begin{abstract}
In this study, polyetheretherketone (PEEK) materials coated with various ratios of two kinds of antibiotic agents (ampicillin and/or vancomycin salts) were prepared. A modified 3D printer based on fused deposition modeling was employed to prepare PEEK disks. Coating ampicillin and/or vancomycin salts onto the PEEK disks was carried out using the biodegradable poly (lactic-co-glycolic acid) (PLGA) polymer as a binder and a control unit for the drug release in the buffer solution. The effects of various rations of ampicillin and/or vancomycin salts in the PLGA polymer on the PEEK substrates, the release profiles of various drugs, and antibacterial activities of the samples were investigated. Temperature of the heated nozzle in a commerical 3D printer was set at $340^{\circ} \mathrm{C}$. After systemic investigations of the qualities of PEEK disks, a diameter of the heated nozzle of $0.6 \mathrm{~mm}$ in the 3D printer was employed for the preparation of PEEK disks. Results of drug release profiles from samples into buffer solution show that the antibacterial activities of samples can continue up to 28 days. In the inhibition zone test of samples, the release amounts of antibiotic agents from the PEEK samples can inhibit S. aureus with activity of over $40 \%$ in 30 days tests and most of them can have inhibition activities of higher than $60 \%$ during the test. These results showed that a simple and low-cost 3D printing method for the preparation of PEEK/antibiotic agents/PLGA samples can have further applications in biomedical-related technology.
\end{abstract}

Keywords: Polyetheretherketone; 3D-printing; fused-deposition modeling; ampicillin; vancomycin; antibacterial property

\section{Introduction}

Industrial 3D printing technology has become more and more important due to its fast manufacturing time, low cost, and the un-moldable printing [1]. According to the report proposed by Wohlers Associates [2], industrial applications in 3D printing technology approached around 12.5 billion US dollars in the year of 2018 and it will attain around 21.2 billion US dollars in the year of 2020. Major applications in 3D printing technology include mechanical manufacturing, aerospace industry, food, and biology-related technologies. In the applications of biology-related technology, 
such as bone and tissue engineering, 3D printing technology has been employed because of the clinical requirements of orthopedic suffers [3-5]. In the clinical requirements of bone and tissue engineering, $3 \mathrm{D}$ and porous bone or tissue supports with suitable surface areas for attachments and growths of cells are often used in the treatments of complex musculoskeletal wounds [3-5]. Several possible manufacturing methods such as electrospinning, solvent casting/particulate leaching, freeze drying, and gas forming have been reported for the production of these 3D supports [6,7]. However, it is difficult for these methods to obtain 3D supports with uniform pore size distribution and suitable shapes that meet the clinical requirements. In contrast, complex 3D supports with uniform pore size distribution can be easily manufactured using 3D printing technology. Three-dimensional printing technology based on the selective laser sintering was employed to prepare porous $\mathrm{Ti}_{6}-\mathrm{Al}_{4}-\mathrm{V}$ supports with mechanical property similar to the bone tissue [6]. Three-dimensional printing technology based on the fused deposition modeling for the preparation of supports (e.g., low-crystalline polymers such as poly lactic acid (PLA)) in the application of bone-tissue engineering was also reported in the literatures $[4,5,7]$. Traditional Ti-based supports (e.g., $\mathrm{Ti}_{6}-\mathrm{Al}_{4}-\mathrm{V}$ ) have been employed for the treatments of musculoskeletal wounds [8]. Even the metal-based supports have been applied in orthopedic suffers; major problems for the treatments of musculoskeletal wounds using Ti-based supports are the metal ions released from these metal supports caused by corrosion in the human body and the mismatch elastic moduli between the human bone (7-30 GPa) and the metal supports ( $110 \mathrm{GPa}$ for the Ti-based metal supports) [5,9]. Low-crystalline polymer supports applied in the bone-tissue engineering cannot maintain long-term stability in the human body if these supports are used in the treatments of orthopedic suffers. Recently, an interesting polymer called polyetheretherketone (PEEK) has been proposed in the applications of bone and tissue engineering $[5,9]$. PEEK is a semi-crystalline polymer with good elastic modulus (3-4 GPa), which is similar to that for human bone (7-30 GPa) $[5,9]$. Similar elastic moduli for the PEEK material and the human bone can minimize the effect of stress shielding, which can result in prolonging implant lifespan and in better mechanical compatibility than the Ti-based metal supports [10]. Good thermal and chemical properties for PEEK are also reported in the literatures [9-13] and make it a good candidate for orthopedic applications. Although the PEEK is a good candidate that can be applied in the orthopedic implant, relative high melting $\left(\sim 340^{\circ} \mathrm{C}\right)$ and glass-transition $\left(\sim 190^{\circ} \mathrm{C}\right)$ temperatures for PEEK compared with traditional low-crystalline polymers used in commerical 3D printers based on fused modeling result in difficulty in their fast manufacture [5]. Major 3D printing technologies used for the printing of these PEEK supports are the powder bed, inkjet head 3D printing (3DP) [12], or the selective laser sintering (SLS) [13]. Generally, the sintering (surface sintering) or binding of high-melting powder materials (e.g., PEEK) with low melting binders was employed to prepare the complex 3D supports by using the 3DP technology [12]. However, complex operation process is thus necessary to be employed in the applications of printing PEEK samples with the 3DP method. Another 3D printing technology based on the SLS method is a rapid method for the preparation of complex 3D supports. It uses a focused high-energy source (such as laser energy source) to make metal or PEEK materials melt and to rebuild a 3D support with melting metal or PEEK material in the 3D printer. However, high equipment costs and high energy requirements of 3D printers based on the SLS method are its major problems. Although some possible printing parameters for commerical 3D printers based on the fused deposition modeling were reported for the preparation of PEEK disks [5], the failure percentage for PEEK samples prepared using the commerical $3 \mathrm{D}$ printer based on fused deposition modeling is still too high (failure percentage of around $20 \%$ ). Optimal printing parameters and the design of a traditional 3D printer have to be remodified in order to obtain fast and precise printing for the preparation of the PEEK supports. Even if these complex 3D supports for bone or tissue engineering applications can be obtained using a 3D printer, another important issue for the clinical requirement of orthopedic suffers is its antibacterial property. In the clinical treatment of orthopedic suffers, implant-associated infection is a major complication in orthopedic surgery. According to the report proposed by Bakhshadeh et al. (2017) [14], infection rates of orthopedic surgery are in the range of $1-5 \%$. It increases exponentially for immunocompromised 
patients. The possibility of infection for orthopedic surgery after revision surgery can increase to as high as $5-40 \%$, which indicates that prevention of implant-associated infection is important. Local injection of high doses of antibiotic agent such as ampicillin sodium salt into the human body is thus necessary to prevent implant-associated infection. However, it may result in the waste of antibiotic agents injected into the human body. Suitable antibacterial activity of these implants may reduce the waste of usage of antibiotic agents using local injection. Generally, the recovery time for a complex orthopedic suffer is around one month. Stable local concentration of antibiotic agents near the implant in a human body of higher than the minimum inhibition concentration (MIC; 90) is thus necessary for clinical requirements. Bakhshadeh et al. (2017) [14] prepared the porous Ti-based implants with surface coating of silver ions and vancomycin salt with chitosan/gelatin applied by electrophoretic deposition in order to improve the antibacterial behaviors of Ti-based implants. The antibacterial behaviors of their samples can continue around 21 days using the release of silver ions and vancomycin salt into the buffer solution. Although the antibacterial behavior of metal ions (e.g., silver, zinc, or ferrous ions) or natural compounds (e.g., Chlorogenic acid) were employed with the implants in the literature [14-19], release of these metal ions in the human body may result in safety issues in the human body. In our previous study, the $\mathrm{ZnO}$ nanotube arrays grown on the PEEK disks with direct absorption of the antibiotic agent (vancomycin or am ampicillin sodium salt) were employed in order to test their antibacterial behavior and to reduce the cytotoxicity of silver ions [5]. The results showed that the concentrations of antibiotic agents released from our samples into the buffer solution were higher than MIC 90 for Staphylococcus aureus (S. aureus) and can continue up to $100 \mathrm{~h}$ (around 5 five days). Even though the PEEK samples with suitable antibacterial property were reported, their antibacterial behaviors are not enough to meet the clinical requirements (around one month) of orthopedic suffers. Optimal printing parameters for the preparation of PEEK substrates using 3D printing technology based on the fused deposition modeling are not enough for future industrial or clinical applications. Long-term antibacterial behaviors of these implants have to be developed in order to decrease the possibility of implant-associated infection. Therefore, series systemic investigations for the printing of PEEK disks with various possible designs/parameters (diameter of heated nozzle, design of metal tube, and temperature set in the heated nozzle) for our 3D printer (Black Magic 3D Prusa i3) were employed in order to obtain PEEK samples for further applications. Various ratios of ampicillin sodium salt and/or vancomycin sodium salt mixed with the hydrolysis PLGA polymer were coated onto the 3D-printed PEEK disks using dip coating. In vitro antibiotic agent release profiles from the surfaces of PEEK disks into the buffer solutions and their bacterial inhibition tests on the organisms were carried out in this study to evaluate the antibacterial properties of the PEEK disks.

\section{Materials and Methods}

\subsection{Chemicals and Apparatus}

Ampicillin sodium salt $\left(\mathrm{C}_{16} \mathrm{H}_{18} \mathrm{~N}_{3} \mathrm{NaO}_{4} \mathrm{~S}\right.$, purity $>98 \%$ ), PLGA (weight percent ratio of lactide:gycolide $=50: 50$, purity $>98 \%$ ), vancomycin hydrochloride $\left(\mathrm{C}_{66} \mathrm{H}_{75} \mathrm{Cl}_{2} \mathrm{~N}_{9} \mathrm{O}_{24} \cdot \mathrm{HCl}\right.$, purity $>98 \%$ ), and the phosphate buffer saline (PBS) solution with the purity of greater than $99.9 \%$ were provided from Aldrich Co. The organism used for the bacterial inhibition tests was Staphylococcus aureus (S. aureus, ATCC6538R) provided from the Bioresource Collection and Research Center (BCRC, Taiwan). The Nutrient Broth (NB; beef extract $3 \%$, peptone $5 \mathrm{~g}$ ) was used for tests of bioactivities of samples on S. aureus. PEEK wire with the diameter of $1.75 \mathrm{~mm}$ for the 3D printing was provided by Huaian Ruanke Trade Co. (Jiangsu, China). A 3D printer (Black Magic 3D Prusa i3, Shanghai, China) was employed for modifications and tests of various printing parameters for PEEK samples. A scanning electron microscope (Hitachi, S-3000N, Tokyo, Japan) with accelerating voltage of $15 \mathrm{kV}$ and working distance of $15 \mathrm{~mm}$ was employed to analyze the surface and cross-sectional images of PEEK samples. A UV-Visible spectrophotometer (UV-Vis, Varian CARY50) and HPLC (Pu-2080, Tokyo, JASCO Co.) 
with the SYMMETRY $C_{8}$ column $(4.6 \times 250 \mathrm{~mm}$, Shim-pack, VP-ODS, Tokyo, Japan $)$ was used to determine the concentration of antibiotic agents in the buffer solution bath.

\subsection{Preparation of PEEK/PLGA/Antibiotic Agent Samples}

After we found the optimal designs for our 3D printer, we tried to prepare PEEK substrates with suitable antibacterial abilities and tested their antibacterial abilities. Due to the difficult direct absorption of antibiotic agents (ampicillin salt or vancomycin hydrochloride salt) on PEEK samples [5], it is necessary to mix these antibiotic agents with the hydrolysis polymer (PLGA) to maintain stable antibacterial performances of these PEEK disks. Various amounts of antibiotic agents (pure ampicillin sodium salt; pure vancomycin hydrochloride; and the weight percentages of ampicillin:vancomycin = 25:75, 50:50, and 75:25) with the PLGA as the binder were mixed well. The precursor solution with the total weight percentage of $12.5 \%$ for antibiotic agents and $87.5 \%$ for PLGA was dissolved in an acetone solution (purity $>99 \%$ ) with magnetic stirring at around $30 \mathrm{~min}$, which was the same as that done with the coating parameter reported by Chen et al. (2012) [20]. Coating of PLGA/antibiotic agents onto the PEEK disk was employed using the dip coating. This coating process was repeated at least five times in order to cover the surface of PEEK disk with the PLGA/antibiotic agents. Detailed procedures can be found in the literature $[4,20]$. After the coating of antibiotic agents onto the sample surface, the PEEK disks were kept in a clean container in order to avoid any possible influence from other chemicals or organisms.

\subsection{In Vitro Drug Release Analysis}

In vitro drug release rate tests from the PEEK disks into the buffer solution were carried out in order to evaluate their possible antibacterial behaviors in the buffer solution bath. Similar approaches have been reported in the literature [4,20]. A phosphate buffer solution $(\mathrm{pH} 7.4,30 \mathrm{~mL})$ as the medium was used for the tests of drug release behaviors from the PEEK disks into the solution bath. Samples were incubated in phosphate buffer saline at the temperature of $37^{\circ} \mathrm{C}$ with the wavering rate of $30 \mathrm{rpm}$. The amounts of antibiotic agents released from the samples into the phosphate buffer solution were analyzed with the time interval of $1 \mathrm{~h}$ (short-term tests) and $24 \mathrm{~h}$ (long-term tests) using the UV-Visible spectrophotometer (UV-Vis, Corona, CA, USA) and also checked with the HPLC (Pu-2080, JASCO Co., Tokyo, Japan) with the SYMMETRY $C_{8}$ column $(4.6 \times 250 \mathrm{~mm}$, Shim-pack, VP-ODS, Tokyo, Japan). The phosphate buffer solution $(25 \mathrm{~mL}$ ) was replaced every test in order to avoid the influence of saturated concentrations of antibiotic agent in the buffer solution. The detected wavelengths for ampicillin and vancomycin salt were $220 \mathrm{~nm}$ and $280 \mathrm{~nm}$ using the UV-Vis spectrometer, respectively. The operation parameters for the analysis of amounts for antibiotic agents in the buffer solution using HPLC are the same as in our previous study [4]. The calibration curves for the antibiotic agents in the phosphate buffer solution (all the correction coefficients $>0.99$ ) were made for the determination of unknown concentrations of the antibiotic agents in the buffer solutions.

\subsection{Test of Bioactivity of Samples}

The test of the bioactivity of samples on the organism has been discussed in the literature $[4,18,20]$. The detail procedures were the same as those reported by Chen et al. (2012) [20]. Here, we show brief descriptions: $200-\mu \mathrm{L}$ samples with $S$. aureus inoculum were cultured in $5 \mathrm{~mL}$ of NB and grown in $12 \mathrm{~h}$ at $37^{\circ} \mathrm{C}$ with the constant shaking rate of $220 \mathrm{rpm}$. Finally, the concentration of bacterial suspensions was adjusted to around $10^{8}$ colony-forming unit (CFU)/mL. The antibiotic disk diffusion method for the S. aureus in the agar containing NB was carried out in Petri dishes in order to evaluate antibacterial properties of these samples; $200 \mu \mathrm{L}$ of solutions containing organisms with the bacterial concentration of $10^{8} \mathrm{CFU} / \mathrm{mL}$ were pipetted and seeded onto the agars in the Petri disks for the tests. The inhibition zones were measured every $24 \mathrm{~h}$ with incubation at $37^{\circ} \mathrm{C}$. A calibration curve for the inhibition zone of organism was also determined by the standard concentrations of antibiotic agents $(1,10,100$, and $1000 \mu \mathrm{g} / \mathrm{mL})$. The release concentrations of antibiotic agents were then determined by 
interpreting the curve. The bioactivity of the antibiotic agent on organism (S. aureus) was calculated using the following equation:

$$
\text { Bioactivity }(\%)=\frac{\text { diameter of sample inhibition zone }}{\text { diameter of maximum inhibition zone }}
$$

\section{Results and Discussion}

For 3D printing technology, the most widely used printing method is the extrusion method [1]. Detailed mechanisms for 3D printing technology were reported in the literature [1]. In a 3D printing system based on the extrusion method, also named fused deposition modeling, a gear system drives an amorphous or low crystallite polymer filament into the heated nozzle assembly for extrusion. Several possible materials such as PLA, poly-vinyl alcohol, or ethyl-vinyl acetate have been employed as the base polymers in the applications of 3D printing technology [1]. In order to inject the polymer filaments into heated nozzle smoothly and to avoid a high temperature set in the heated nozzle from damaging the gear system, a metal tube with length of around several centimeters is used to connect the heated nozzle and the gear system. Melted polymer filaments dropped from the heated nozzle form as the stalagmites. Using a control unit of the 3D printer, a 3D object can be built using layer-by-layer deposition. An important parameter for 3D printing is the temperature set in the heated nozzle of a 3D printer. Materials used in a commercial 3D printer are the low-crystalline polymers (e.g., PLA), of which the glass transition temperature $\left(T_{g}\right)$, crystallization temperature $\left(T_{c}\right)$, and melting temperature $\left(\mathrm{T}_{\mathrm{m}}\right)$ are lower than those for PEEK. Therefore, the temperature of the heated nozzle has to be set at a higher temperature than the original design for commerical 3D printers (around $220^{\circ} \mathrm{C}$ ). In our previous study [5], the crystallization and melting temperatures of around $292^{\circ} \mathrm{C}$ and $340{ }^{\circ} \mathrm{C}$ for PEEK were reported. It indicates that the temperature of heated nozzle has to be set higher than $340{ }^{\circ} \mathrm{C}$. However, at around $600{ }^{\circ} \mathrm{C}$, PEEK will decompose accord to the results proposed by Ajeesh et al. (2015) [21]. Therefore, two temperatures of the heated nozzle of around $340^{\circ} \mathrm{C}$ and $360^{\circ} \mathrm{C}$ were set, respectively, in order to make the PEEK filaments melt in the heated nozzle and drop in the form of stalagmites for the building of a 3D object. The temperature of holder was also set at $45^{\circ} \mathrm{C}$, which was the same as in our previous study [5]. Figure 1 shows the 3D printing results of PEEK samples with a different temperature set in the heated nozzle.

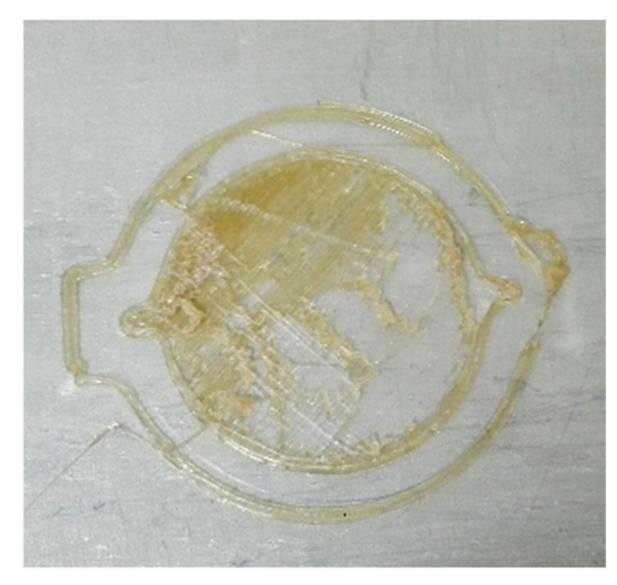

(I)

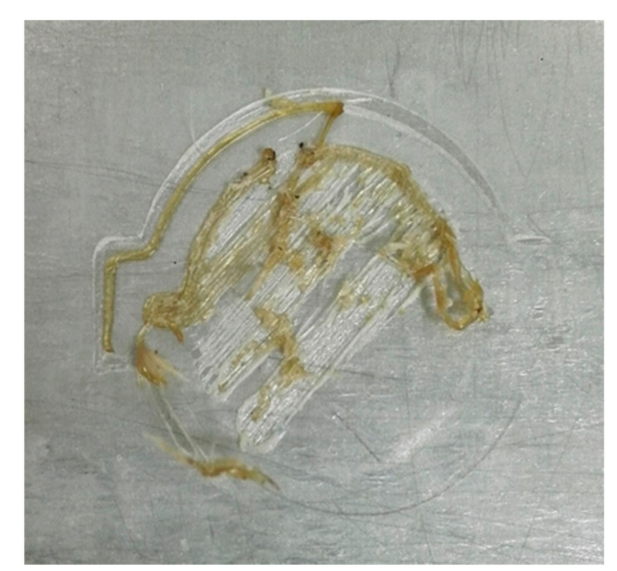

(II)

Figure 1. Printing results of polyetheretherketone (PEEK) samples with temperatures of (I) $340^{\circ} \mathrm{C}$ and (II) $360^{\circ} \mathrm{C}$ in the heated nozzle at the initial printing stage.

From the results shown in Figure 1, poor qualities of 3D printing PEEK samples were observed. Some dark parts in the PEEK sample were observed with the temperature of the nozzle kept at $360^{\circ} \mathrm{C}$, which indicated that the carbonization of the PEEK sample occurred when the temperature of the 
nozzle was kept at $360^{\circ} \mathrm{C}$. For the temperature of the nozzle kept at $340{ }^{\circ} \mathrm{C}$, although no carbonization of the PEEK sample was observed, the failure percentage of around $20 \%$ for the PEEK objects was still observed. It indicated that the heated nozzle or the metal tube must be remodified. After we carefully examined the structure of the heated nozzle and the metal tube connected between the heated nozzle and the gear system, the major problem for a high failure percentage of 3D printing objects was the diameter of the heated nozzle. The original diameter for the heated nozzle loaded in the commerical 3D printer is $0.4 \mathrm{~mm}$. However, a higher viscosity of PEEK compared with that for PLA made the PEEK material block in the heated nozzle and resulted in the high failure percentage of a 3D object. The loading of the metal tube has to be remodified in order to inject the polymer filaments into the heated nozzle smoothly.

Figure 2 shows comparisons of the metal tube and heated nozzle for the original and new designs in our own 3D printer. Increasing the volume of the heated nozzle can decrease the possibility of a jam for PEEK filaments in the heated nozzle. According to the results proposed by Berretta et al. (2017) [22], the diameter of the heated nozzle is very important for the 3D printer based on the fused deposition modeling. A small diameter of the heated nozzle cannot make the PEEK move freely and easily form since the semi-liquid PEEK blocked the heated nozzle although the temperature of the heated nozzle was set higher than its melting point. Semi-liquid type PEEK then damages the heated nozzle for the 3D printer. In order to obtain the optimal diameter of the heated nozzle, various diameters of the heated nozzles, $0.4 \mathrm{~mm}, 0.6 \mathrm{~mm}, 0.8 \mathrm{~mm}$, and $1.0 \mathrm{~mm}$, were tested. Diameters of the heated nozzle at $0.8 \mathrm{~mm}$ and $1.0 \mathrm{~mm}$ could not generate stalagmites at the heated nozzle. Poor 3D printing was observed using diameter $0.4 \mathrm{~mm}$ for the heated nozzle in our own 3D printer. Therefore, a diameter of $0.6 \mathrm{~mm}$ for the heated nozzle in our own 3D printer was employed for the preparation of PEEK samples. The part of the metal tube with a diameter of $2 \mathrm{~mm}$ connected with the heated nozzle can also inject PEEK filament smoothly into the heated nozzle compared with the original design of commerical 3D printer. Detailed comparisons of the original and new designs are shown in Figure 2. After we modified the designs of the heated nozzle and the metal tube, we got good PEEK disks using our $3 \mathrm{D}$ printer.

Original

$0.4 \mathrm{~mm}$

(1)

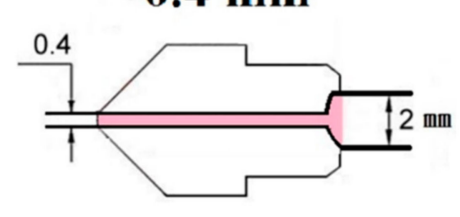

(2)

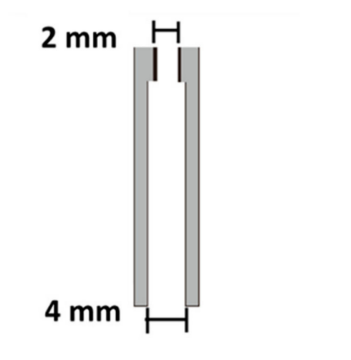

\section{New Design}

\section{$0.6 \mathrm{~mm}$}
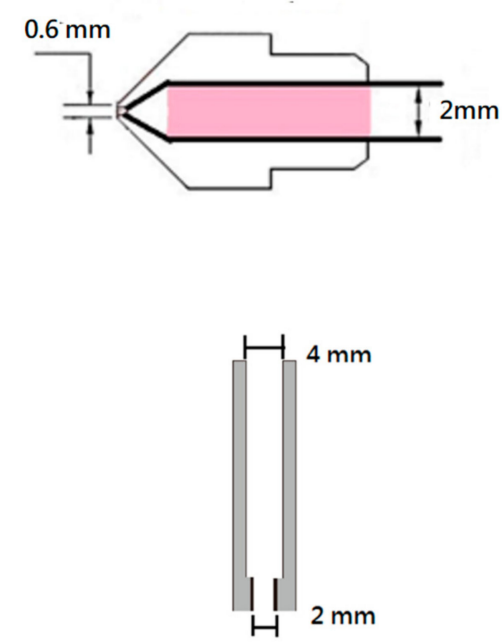

Figure 2. Summary of the original and new designs for the heated nozzle (1) and metal tube (2) used in this study.

Figure S1 in the Supplementary Materials shows the images of PEEK disks using the original and modified designs in our own 3D printer. In Figure S1I, a poor PEEK disk was obtained using the 
commerical 3D printer. After we modified the parameters and designs of our 3D printer, a uniform PEEK disk can be obtained (see Figure S1II). Figure S2 also shows the surface and cross-sectional SEM images of the PEEK disks prepared using our modified 3D printer. A uniform PEEK sample without pinholes or voids can be observed in Figure S2I. In Figure S2II, the fiber thicknesses of 600-900 nm were observed for PEEK disk. It indicates that our modifications are suitable for the production of PEEK samples using the 3D printer based on the fused modeling method. Therefore, these new designs were employed to prepare the PEEK disks for further tests. For the test of the antibacterial performances of PEEK samples, the target of this study is to obtain a PEEK sample with suitable antibacterial ability of around one month. Due to the poor absorption of antibiotic agents on the PEEK sample, PLGA was used as the binder of antibiotic agents and the control-release unit of antibiotic agents released into the buffer solution. When the PLGA contacts buffer solutions, the PLGA will decompose by hydrolysis of its ester linkages and produce lactic acid and glycolic acid and the antibiotic agents coated at the PEEK sample then release into the buffer solution. Table 1 shows the coating parameters for the various ratios of ampicillin/vancomycin on the PEEK substrates using the PLGA, which are the weight percent of ampicillin/total antibiotic agents of around $0 \%, 25 \%, 50 \%, 75 \%$, and $100 \%$. Drug release profiles for vancomycin and ampicillin from the samples into the buffer solution as a function of time in the 24-h test are shown in Figure S3I,II, respectively. From the results shown in Figure S3, both the drug release profiles of vancomycin and ampicillin were not stable and showed a peak profile for all samples in the first $6 \mathrm{~h}$ when the test began. The maximum drug release concentration for vancomycin and ampicillin of around $54 \mathrm{mg} / \mathrm{L}$ and $46 \mathrm{mg} / \mathrm{L}$ for samples (B) and (D) were observed, respectively. When the times for the tests were in the range of 7-24 h, the drug release profiles for ampicillin showed a stable increase with time for samples (B)-(E). However, the vancomycin release profiles for samples (C) and (D) decreased as a function of time.

Table 1. Coating parameters for ampicillin/vancomycin onto the PEEK substrates.

\begin{tabular}{ccccc}
\hline \multirow{2}{*}{ Sample } & $\begin{array}{c}\text { PLGA } \\
\text { Weight (mg) }\end{array}$ & $\begin{array}{c}\text { Ampicillin } \\
\text { Weight (mg) }\end{array}$ & $\begin{array}{c}\text { Vancomycin } \\
\text { Weight (mg) }\end{array}$ & $\mathbf{W}_{\text {Amp }}$ \\
\cline { 1 - 1 } & & & $\mathbf{W}_{\text {Amp+Van }}$ & \\
(A) & 875.6 & 0 & 125.5 & 0 \\
(B) & 875.5 & 33.0 & 94.0 & 26.0 \\
(C) & 876.0 & 63.3 & 63.4 & 50.0 \\
(D) & 876.3 & 94.2 & 32.4 & 74.3 \\
(E) & 876.5 & 125.3 & 0 & 100.0 \\
\hline
\end{tabular}

Peak release profiles for antibiotic agents in buffer solutions may be due to the physical absorption of the drug on the surface of the PLGA layers. When the samples contacted the buffer solution, the antibiotic agent weakly absorbed on the PLGA layer was quickly released into the solution. Therefore, a peak profile of the drug in the buffer solution shown in Figure S3I,II was observed for all samples. From the results shown in Figure S3, we found that the release profiles for vancomycin salt in the buffer solution increased as a function of time for samples (A) and (B) in the period of 7-24 h during the test. The release profiles for vancomycin in the buffer solution for samples (C) and (D) increased as a function of time in the period of 6-12 h. After that, they decreased as a function of time. Please note loading amounts of vancomycin of around $50 \%$ and $25 \%$ for samples (C) and (D) were employed, respectively, which indicated that the amount of physical absorption of vancomycin in samples (C) and (D) may run out after $12 \mathrm{~h}$ during the test. Therefore, they decreased as a function of time after the $12-\mathrm{h}$ test. For the results shown in Figure S3II, the drug release profiles of samples (B)-(E) increased with the loading amount of ampicillin on the samples. However, the release profile for the ampicillin for sample (D) showed the maximum value compared with other samples at the same time. A possible reason is due to the number of acidic function groups and the molecular weight of ampicillin and vancomycin [23-25]. The values of PKa for vancomycin and ampicillin are 2.99 and 3.24, respectively [23]. Gentile et al. (2014) [26] also reported that the acidic surrounding media accelerates the degradation of PLGA due to 
autocatalysis. Theoretically, the amount of drug release profile for vancomycin with the degradation of PLGA is higher than that for ampicillin due to it having a lower PKa value. Therefore, the amount of drug release for sample (A) is the highest due to the maximum amount of vancomycin loaded on sample (A). However, they showed different results. Therefore, the short-term release profiles and the cumulative release profiles for total amounts of antibiotic agents (vancomycin + ampicillin salts) into the buffer solution for samples (A)-(E) are shown in Figure 3I,II), respectively. Total amounts of drug released from the mixtures of ampicillin and vancomycin (samples (B)-(D)) in the buffer solution were higher than pure vancomycin (sample (A)) and ampicillin (sample (E)). From the results shown in Figure 3I, the amount of drug in the buffer solution for sample (A) is higher than that for sample (E), which agrees well with the pKa values for vancomycin and ampicillin $[25,26]$. A lower pKa value results in faster degradation of PLGA and releases more drug into the buffer solution. With the decrease in the loading amount of vancomycin in the samples (samples (B)-(D)), the released amount of drug in the buffer solution would also decrease if only the Pka factor influences the degradation of PLGA. However, sample (D) had the maximum released amount of antibiotic agent in the buffer solution, which indicated that other reasons affected the degradation of PLGA. The molecular weight for ampicillin $(373.39 \mathrm{~g} / \mathrm{mol})$ is much smaller than that for vancomycin $(1449.25 \mathrm{~g} / \mathrm{mol})$. Total moles of the antibiotic agents for sample (D) are larger than samples (C) and (B). Although the pKa value for vancomycin reported is lower than that for ampicillin, the difference between the values of $\mathrm{pKa}$ for vancomycin and ampicillin is not too much larger. Because sample (D) had the highest mole value for antibiotic agents compared to the other two samples (samples (B) and (C)), it indicated that the number of acidic groups for sample (D) was higher than other two samples, which resulted in the fast degradation of PLGA and resulted in larger amounts of drug released from sample (D) into the buffer solution compared with other samples. Figure 3II also showed cumulative release profiles for the total amount of antibiotic agents released from samples (A)-(E) into the buffer solution. Around $7-10 \%$ of the total amount of antibiotic agents released from samples (A)-(C) and (E) into the buffer solution was observed in the one-day test. Around 30\% of the total amount of antibiotic agents released from sample (D) into the buffer solution was observed in the one-day test, which indicated that the long-term antibacterial performance for sample (D) may be worse than the other samples. In order to test long-term antibacterial performances of the samples, Figure 4I,II shows the long-term release profiles and the cumulative release profiles for the total amount of antibiotic agents in the buffer solution for samples (A)-(E).

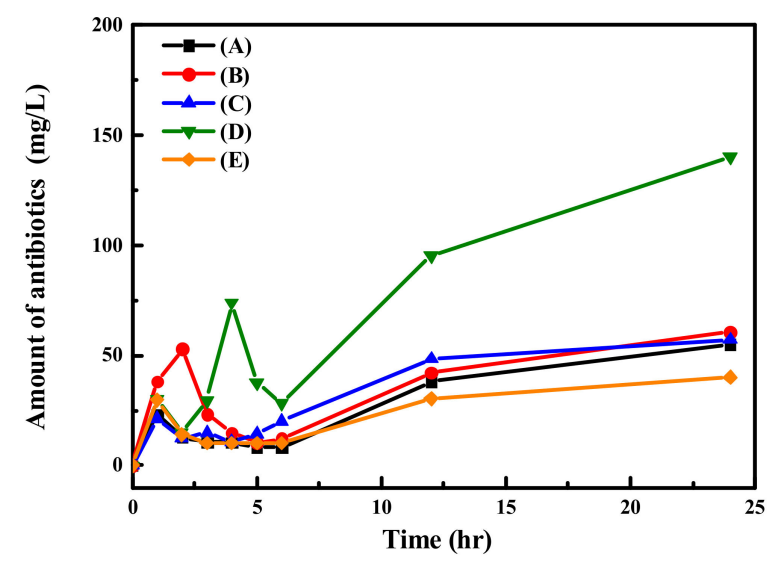

(I)

Figure 3. Cont. 


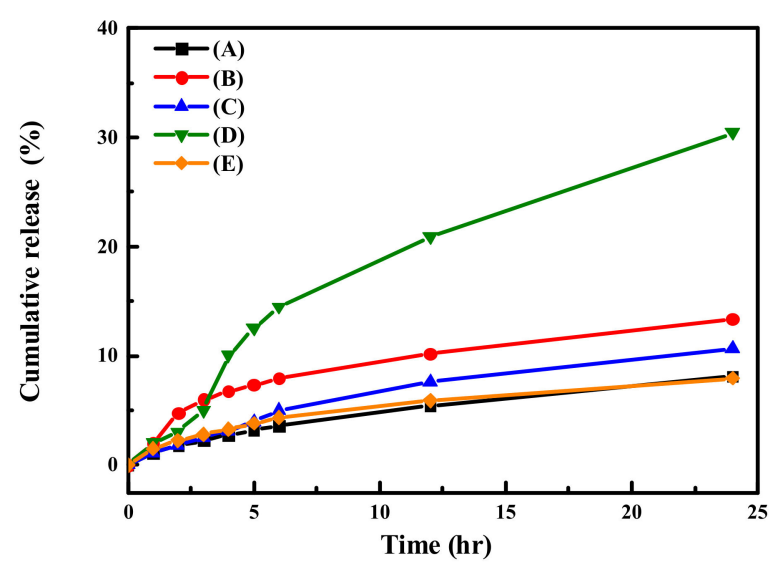

(II)

Figure 3. (I) Short-term release profiles and (II) the cumulative release profiles for the total amount of antibiotic agents released from PEEK into the buffer solution for samples (A)-(E).

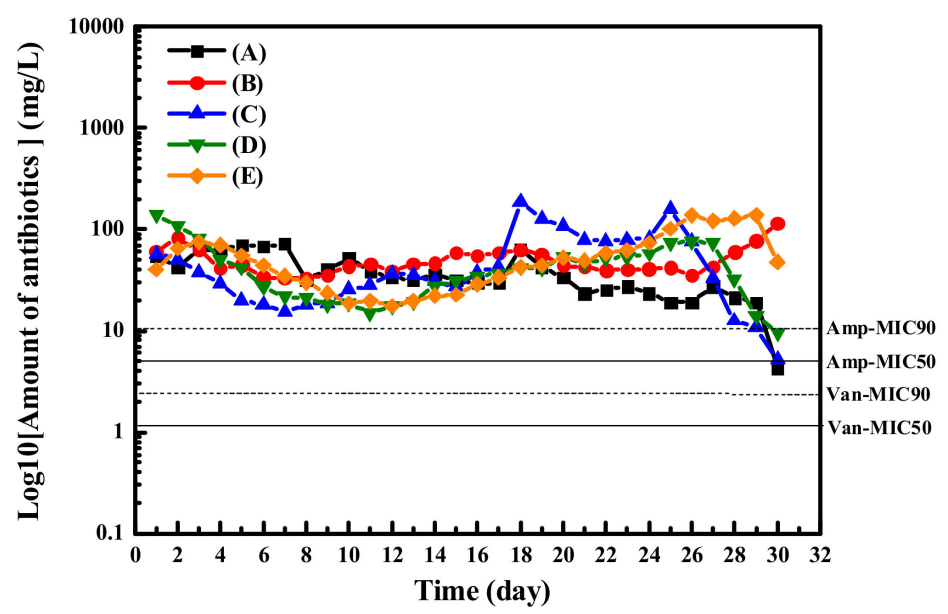

(I)

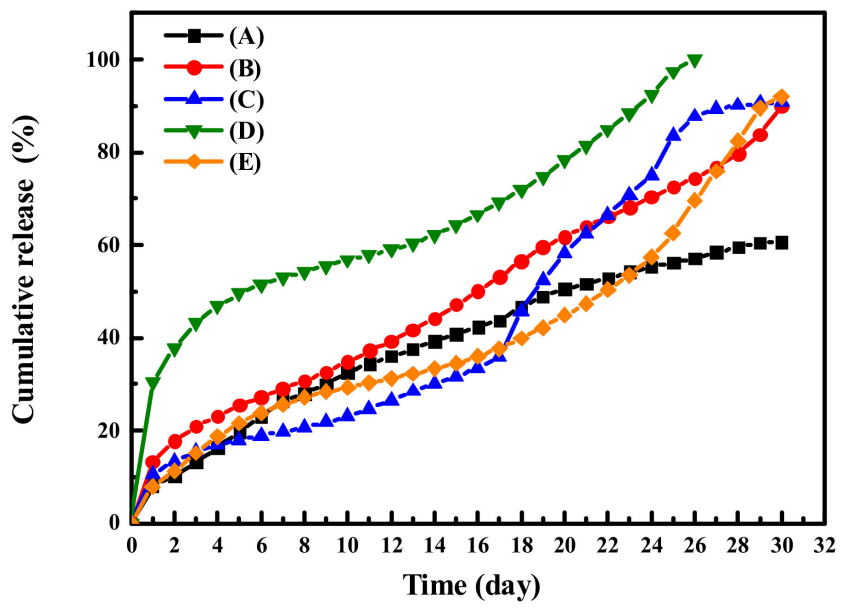

(II)

Figure 4. (I) Long-term release profiles and (II) the cumulative release profile for the total amount of antibiotic agents in the buffer solution for samples (A)-(E). 
Detailed drug release profiles for vancomycin and ampicillin salts in the buffer solution in the 30-day test are also given in Figure S4I,II, respectively. The values of MIC 50 and MIC 90 of S. aureus for vancomycin and ampicillin reported in the literatures are also given in Figure 4I and Figure S4 [27-29]. For the results shown in Figure S4I, the concentrations for vancomycin salts released from samples (A)-(D) in the 30-day test were almost higher than the value of MIC 90 for S. aureus. However, the concentration of vancomycin released from the samples (C) and (D) approached the value of MIC 90 for S. aureus on the 30th and 29th day, respectively. It indicated that the degradations of PLGA for samples (C) and (D) were a little fast compared to those for samples (A) and (D) due to the high number of acidic groups in these samples. The same results can be also found at the ampicillin release profiles for samples (B)-(E) in the buffer solution, as shown in Figure S4II. Figure 4I shows the variations of the total amount of antibiotic agents released from the samples as a function of time. Total concentrations of antibiotic agents released from samples (C) and (D) were in the range of values for MIC 90 on S. aureus for ampicillin and vancomycin on the 27th-30th day tests. The cumulative release profiles for the total amount of antibiotic agents from samples (A)-(E) into the buffer solutions are given in Figure 4II. The released percentages of antibiotic agents from sample (D) into buffer solution approached $100 \%$ at around the 24th day test due to the faster degradation of PLGA. It indicated that the antibacterial property of sample (D) only remained around 24 days and cannot approach the clinical requirement (around one month). Total percentages of antibiotic agents released from samples (A)-(C) and (E) were still lower than 100\%, which indicated that their antibacterial properties may be higher than one month. Samples $(A)-(C)$ and $(E)$ can meet the antibacterial requirements of orthopedic surgery. Therefore, we tested the antibacterial performances for samples (A)-(E) using the disk-diffusion method. Figure 5 shows the bioactivity and the estimation of drug release distributions of antibiotic agents in the Petri disks as a function of time using the calibration curve for the inhibition of $S$. aureus.

From the results shown in Figure 5I-V, the bioactivity of sample (A) (only vancomycin/PLGA coated on the PEEK disk) on S. aureus was kept at $80 \%$ on the first 15 days and decreased to around $20 \%$ for the 15 th to 20 th days. After that, the bioactivity of samples increased to $90 \%$. The results showed the good bioactivity of sample (A) on the first 15 day tests due to the release of vancomycin that weakly absorbed or coated the surface of the PLGA layer. For the period of the 15th-20th day tests, the vancomycin at the sample surface ran out and made a fast decrease in the value of bioactivity of sample (A) on S. aureus. After that, the degradation of the PLGA layer continued and the concentration of vancomycin also increased and maintained its bioactivity on S. aureus up to 30 days. However, the unstable drug-release profile for sample (A) would increase the possibility of infection. For sample (B), a bioactivity of the sample higher than $80 \%$ was observed at the first 20 days and decreased quickly to $10 \%$. For the application of orthopedic surgery, the recovery time of an orthopedic patient is around 28 days. It indicates that its antibacterial property is not enough to meet the antibacterial requirement of orthopedic surgery. However, sample (B) may have good application if the recovery time of a patient is less than 20 days. The drug release behavior and the bioactivity of sample (C) were similar to those of sample (A), but its bioactivity was still higher than sample (A), which indicated that sample (C) had better antibacterial property than sample (A). For samples (D) and (E), their bioactivities could remain at least 18 days and decrease to around $30 \%$ from the 18 th day to the 30th day. It seems that the antibacterial properties of samples (D) and (E) were not good enough to meet the long-term antibacterial property of orthopedic surgery. For the above results, the 3D-printing PEEK sample with the coating of vancomycin (50\%) + ampicillin (50\%) using the PLGA as the control-release unit is good for application in orthopedic surgery. 


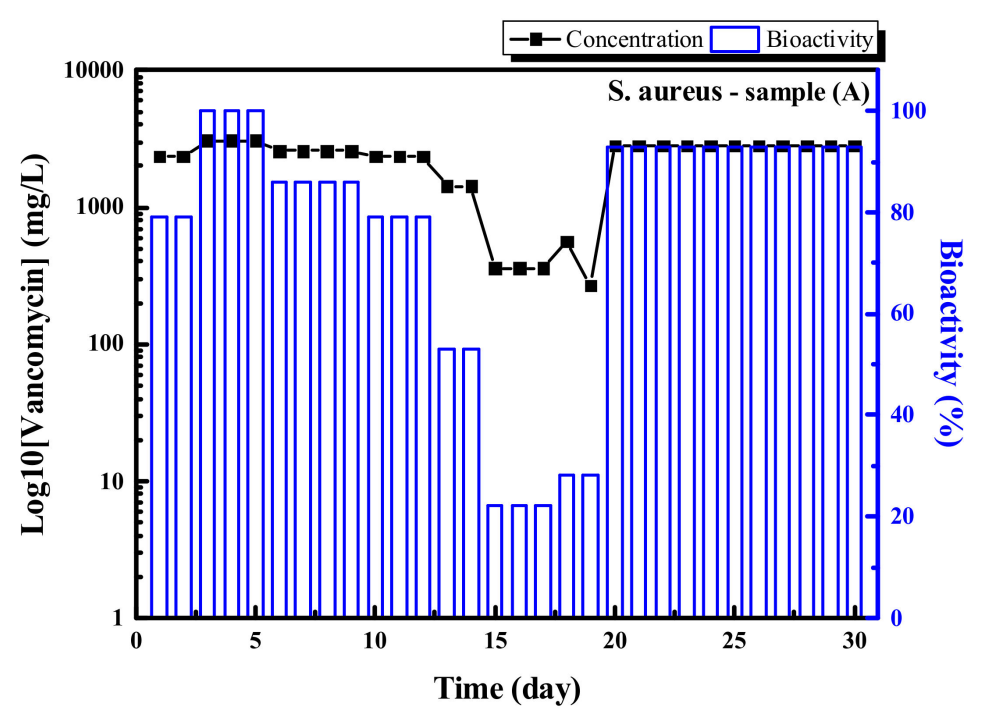

(I)

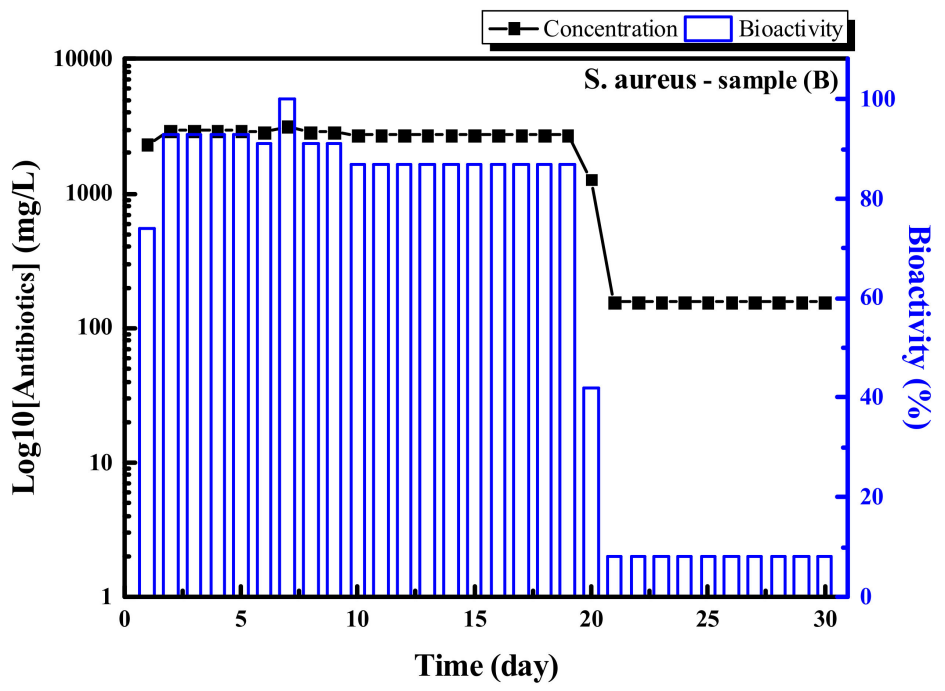

(II)

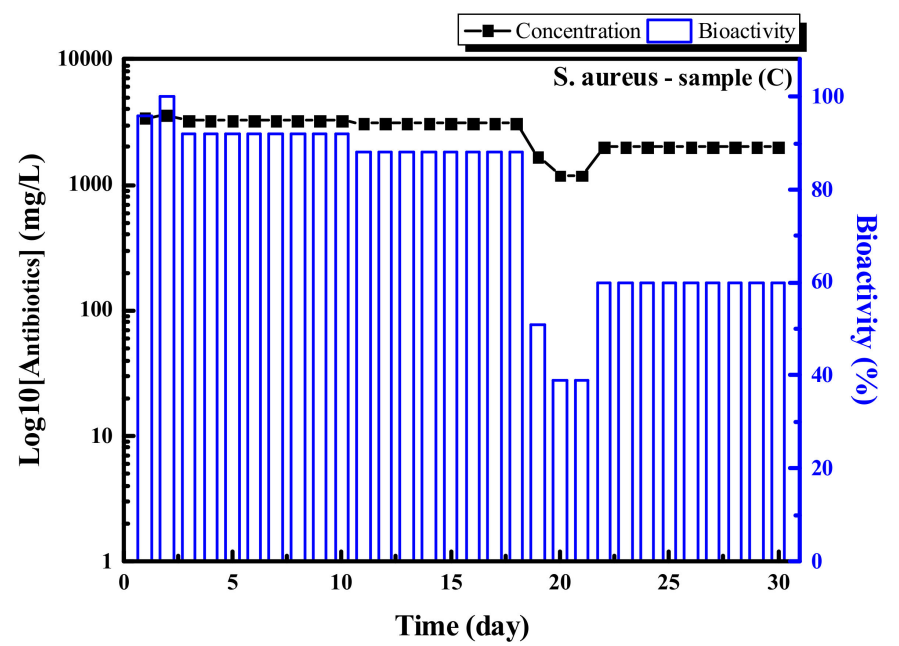

(III)

Figure 5. Cont. 


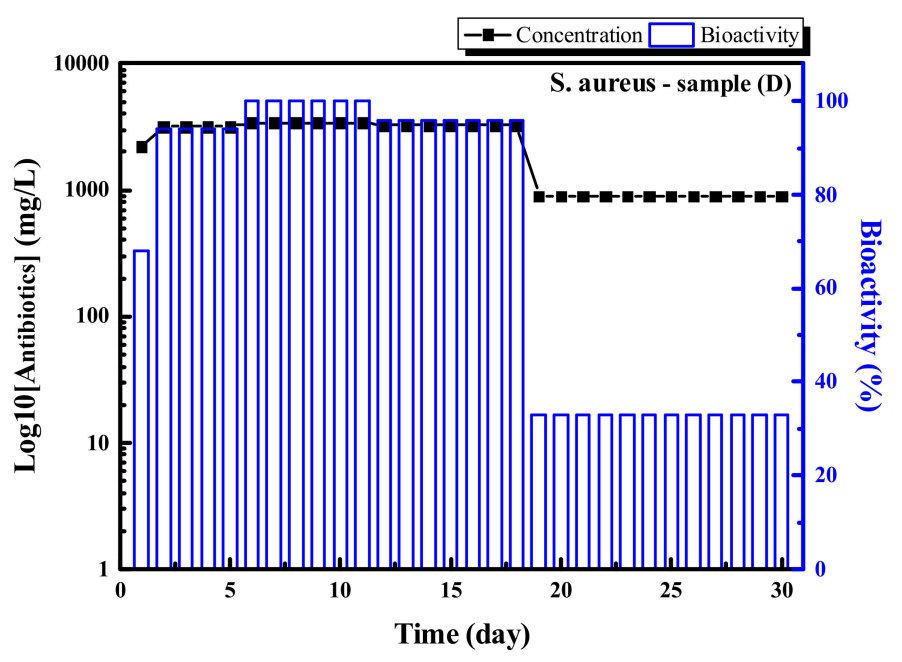

(IV)

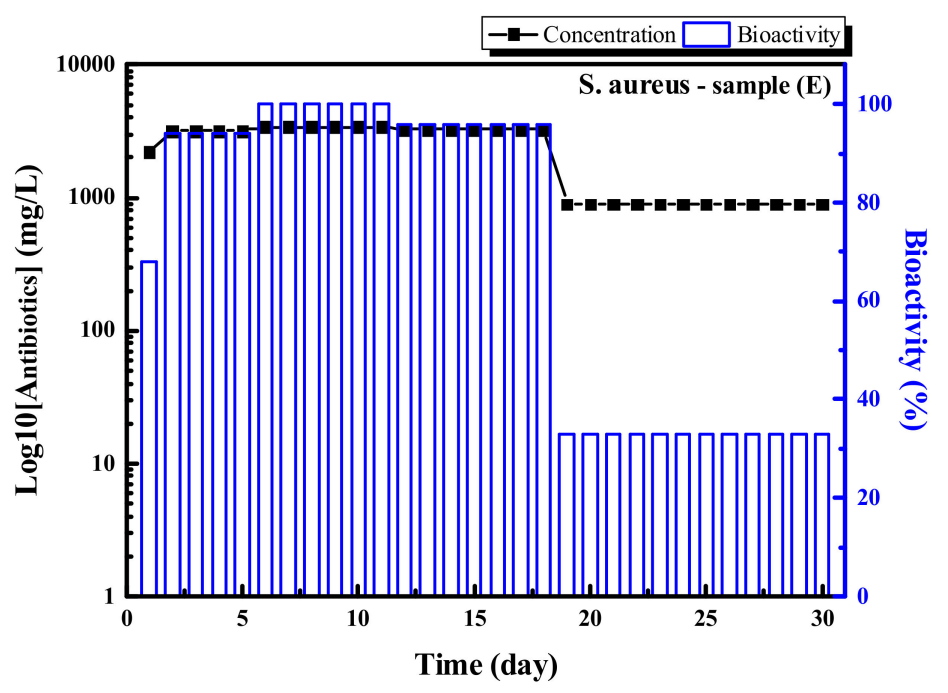

(V)

Figure 5. Bioactivity for S. aureus for (I) sample (A), (II) sample (B), (III) sample (C), (IV) sample (D), and $(\mathbf{V})$ sample $(\mathrm{E})$, respectively.

\section{Conclusions}

In this study, we tried to develop modifications of a commerical 3D printer to prepare the PEEK substrates using the fused deposition modeling method. Then, the PEEK/antibiotic agent samples were prepared using dip coating with various contents of vancomycin/ampicillin salts mixed with PLGA binder in order to obtain the samples with long-term antibacterial properties. Several possible components such as the design of metal tube, the diameter of heated nozzle, and the temperatures set in the heated nozzle were examined. When the temperature of the heated nozzle was higher than $360^{\circ} \mathrm{C}$, a coalification was observed in the PEEK sample, which indicated that the temperature of the heated nozzle had to be set at $340{ }^{\circ} \mathrm{C}$. The diameter of $0.6 \mathrm{~mm}$ for the heated nozzle is better than that of $0.4 \mathrm{~mm}$ when the PEEK materials were used for 3D printing. The part of metal tube with a diameter of $2 \mathrm{~mm}$ connected with the heated nozzle can also inject PEEK filament smoothly into the heated nozzle compared to the original design of a commerical 3D printer. For the drug release profile tests of antibiotic agents in the buffer solution, stable drug release profiles for ampicillin and/or vancomycin from the samples into the buffer solution were observed and made the concentrations of antibiotic agents in the buffer solution higher than the values of MIC 90 on S. aureus within a 30-day 
tests. Bioactivities of the ampicillin and/or vancomycin loaded on the PEEK substrates can maintain at least 28 days. The loading ratio of vancomycin (50\%) and ampicillin (50\%) on the PEEK substrate showed good antibacterial performance compared with other samples. This study showed that a simple and low-cost 3D printing method for the preparation of PEEK/antibiotic agent material can have further applications in biomedical-related technology.

Supplementary Materials: The following are available online at http://www.mdpi.com/2076-3417/10/1/97/s1: Figure S1. Printing results of PEEK sample using the (I) original 3D printer and (II) modification of our 3D printer. Figure S2. (I) Surface SEM images of PEEK sample and (II) cross-sectional images of PEEK sample. Figure S3. Short-term drug-release profiles for (I) vancomycin and (II) ampicillin salts from the samples on the PEEK substrates into the buffer solution. Figure S4. Long-term drug-release profiles for (I) vancomycin and (II) ampicillin salts from the samples on the PEEK substrates into the buffer solution.

Author Contributions: The designs of experiments were carried out by D.W.C. and K.-W.C. The preparation and characterization of PEEK/antibiotic agent/PLGA samples were carried out by M.-H.T. and N.-C.L. The analysis of antibacterial properties of samples was carried out by N.-C.L. and C.-H.C. The writing and correction were made by D.W.C. and K.-W.C., and all authors have given approval to this manuscript. All authors have read and agreed to the published version of the manuscript.

Funding: This work was sponsored by the Chang Gung Memorial Hospital, Keelung grant numbers CMRPD3G0071, CMRPD3G0072, and BMRP948.

Acknowledgments: The authors thank Chang Gung Memorial Hospital, Keelung and Chang Gung University for supporting this study.

Conflicts of Interest: The authors declare no conflict of interest.

\section{References}

1. Norman, J.; Madurawe, R.D.; Moore, C.M.V.; Khan, M.A.; Khariuzzaman, A. A new chapter in pharmaceutical manufacturing: 3D-printed drug products. Adv. Drug Deliv. Rev. 2017, 108, 39-50. [CrossRef] [PubMed]

2. Wholers, T.; Caffrey, T.; Campbell, R.I.; Diegel, O.; Kowen, J. 3D Printing and Additive Manufacturing State of the Industrial Annual Worldwide Progress Report; Wohlers Associate: Fort Collins, CO, USA, 2018.

3. Goole, J.; Amighi, K. 3D printing in pharmaceutics: A new tool for designing customized drug delivery system. Int. J. Pharm. 2016, 499, 376-394. [CrossRef] [PubMed]

4. Chen, C.H.; Yao, Y.Y.; Tang, H.C.; Lin, T.Y.; Chen, D.W.; Cheng, K.W. Long-term antibacterial performances of biodegradable polylactic acid materials with direct absorption of antibiotic agent. RSC Adv. 2018, 8, 16223-16231. [CrossRef]

5. Chen, D.W.; Lee, K.Y.; Tsai, M.H.; Lin, T.Y; Chen, C.H.; Cheng, K.W. Antibacterial applications on staphylococcus aureus using abtibiotic agent/zinc oxide nonorod arrays/polyethylethylketone composite samples. Nanomaterials 2019, 713, 1-15.

6. Sallica-Leva, E.; Jardine, A.L.; Fagagnolo, J.B. Microstructure and mechanical behavior of porous Ti-6Al-4V parts obtained by selective laser melting. J. Mech. Behav. Biomed. Mater. 2013, 26, 98-108. [CrossRef]

7. Grémare, A.; Guduric, V.; Bareille, R.; Heroguez, V.; Latour, S.; L'heureux, N.; Fricain, J.C.; Catros, S.; Le Nihouannen, D. Characterization of printed PLA scaffolds for bone tissue engineering. J. Biomed. Mater. Res. A 2018, 106, 887-894. [CrossRef]

8. Quyang, L.; Zhao, Y.; Jin, G.; Lu, T.; Qiao, Y.; Ning, C.; Zhang, X.; Chu, P.K.; Liu, X. Influence of sulfur content on the bone formation and antibacterial ability of sulfonated PEEK. Biomaterials 2017, 115-126.

9. Kizuki, T.; Matsushita, T.; Kokubo, T. Apatite-forming PEEK with $\mathrm{TiO}_{2}$ surface layer coating. J. Mater. Sci. Mater. Med. 2015, 26, 1-9. [CrossRef]

10. Anguiano-Sanchez, J.; Martinez-Romero, O.; Siller, H.R.; Diaz-Elizondo, J.A.; Flores-Villalba, E.; Rodriguez, C.A. Influence of PEEK coating on Hip implant stress shielding: A finite element analysis. Comput. Math. Methods Med. 2016, 2016, 6183679. [CrossRef]

11. Wu, J.; Li, L.; Fu, C.; Yang, F.; Jiao, Z.; Shi, X.; Ito, Y.; Wang, Z.; Liu, Q.; Zhang, P. Micro-porous polyetheretherketone implants decorated with BMP-2 via phosphorylated gelatin coating for enhance cell adhesion and osteogenic differentiation. Colloids Surf. B Biointerfaces 2018, 169, 233-241. [CrossRef]

12. Parthasarathy, J. 3D modeling custom implants and its future perspectives in craniofacial surgery. Ann. Maxillofac. Surg. 2014, 4, 9-18. [CrossRef] [PubMed] 
13. Schmidt, M.; Pohle, D.; Rechtenwald, T. Selective Laser Sintering of PEEK. CIRP Ann. 2007, 1, $205-208$. [CrossRef]

14. Bakhshandeh, S.; Karaji, Z.G.; Lietaert, K.; Fluit, A.C.; Boel, C.H.H.; Vogely, H.C.; Vermoden, T.; Hennink, W.E.; Weinans, H.; Zadpoor, A.A.; et al. Simultaneous delivery of multiple antibacterial agents from additively manufactured porous biomaterials to fully eradicate planktonic and adherent Staphylococcus aureus. ACS Appl. Mater. Interfaces 2017, 9, 25691-25699. [CrossRef] [PubMed]

15. Felice, B.; Sánchez, M.A.; Socci, M.C.; Sappia, L.D.; Gómez, M.I.; Cruz, M.K.; Felice, C.J.; Marti, M.; Pividon, M.I.; Simonelli, G.; et al. Control degradability of PCL-ZnO nanofibrous scaffolds for bone tissue engineering and their antibacterial activity. Mater. Sci. Eng. C 2018, 93, 724-738. [CrossRef] [PubMed]

16. Kadiyala, U.; Turali-Emre, E.S.; Bahng, J.H.; Kotov, N.A.; VanEpps, J.S. Unexpected insights into antibacterial activity of zinc oxide nanoparticels against methicillin resistant Staphylococcus aureus (MRSA). Nanoscale 2018, 10, 4427-4439. [CrossRef]

17. Agnihotri, S.; Bajaj, G.; Mukherji, S.; Mukherji, S. Arginine-assisted immobilization of silver nanoparticles on $\mathrm{ZnO}$ nanorods: An enhanced and reusable antibacterial substrates with human cell cytotoxicity. Nanoscale 2015, 7, 7415-7429. [CrossRef]

18. Tranquillo, E.; Barrino, F.; Poggetto, G.D.; Blanco, I. Sol-Gel synthesis of silica-based materials with different percentages of PEG or PCL and high chlorogenic acid content. Materials 2019, 12, 155. [CrossRef]

19. Catauro, M.; Tranquillo, E.; Barrino, F.; Blanco, I.; Poggetto, G.D.; Navigllo, D. Drug Release of Hybrid Materials Containing Fe(II)Citrate Synthesized by Sol-Gel Technique. Materials 2018, 11, 2270. [CrossRef]

20. Chen, D.W.; Hsu, Y.H.; Liao, J.Y.; Liu, S.J.; Chen, J.K.; Ueng, S.W.N. Sustainable release vancomycin gentamicin and lidocaine from novel electrospun sandwich-structured PLGA/collagen nanofibers membrane. Int. J. Pharm. 2012, 430, 335-341. [CrossRef]

21. Ajeesh, G.; Bhowmik, S.; Sivakumar, V.; Varshney, L.; Kumar, V.; Abraham, M. Investigation on polyetheretherketone composite for long term storage of nuclear waste. J. Nuclear Mater. 2015, 467, 855-862. [CrossRef]

22. Berretta, S.; Davies, R.; Shyng, Y.T.; Wang, Y.; Ghita, O. Fused deposition modeling of high temperature polymer: Exploring CNT PEEK composites. Polym. Test. 2017, 63, 251-262. [CrossRef]

23. Kumar, H.; Singla, M.; Jindal, R. Solvation behavior of depeptides of alanine in aqueous solution of antibacterial drug ampicillin at different temperature. Thermo. Acta 2013, 571, 28-41. [CrossRef]

24. Levine, D.P. Vancomycin: A History. Clin. Infect. Dis. 2006, 42, S5-S12. [CrossRef] [PubMed]

25. Kang, M.A.; Kang, J.S. Stability test of ampicillin sodium solutions in the accufuser ${ }^{\circledR}$ elastomeric infusion device Using HPLC: UV method. Pharmacol. Pharm. 2012, 4, 462-467. [CrossRef]

26. Gentile, P.; Chiono, V.; Carmagnola, I.; Hatton, P.V. An overview of poly (lactic-co-glycolic) acid (PLGA)-based biomaterials for bone tissue engineering. Int. J. Mol. Sci. 2014, 15, 3640-3659. [CrossRef]

27. Xiao, L.; Mai, Y.; He, F.; Yu, L.; Zhang, L.; Tange, H.; Yang, G. Bio-based green composites with high performance from poly(lactic acid) and surface-modified microcrystalline cellulose. J. Mater. Chem. 2012, 22, 15732-15739. [CrossRef]

28. Sancak, B.; Yagci, S.; Gür, D.; Gülay, Z.; Ogunc, D.; Söyletir, G.; Yalcin, A.N.; Dündar, D.Ö.; Topçu, A.W.; Aksit, F.; et al. Vancomycin and daptomycin minimum inhibitory concentration distribution and occurrence of heteroresistance among methicillin-resistant Staphylococcus aureus blood isolates in Turkey. BMC Infect. Dis. 2013, 13, 583-589. [CrossRef]

29. Sidorenko, S.V.; Rezvan, S.P.; Tikchonova, A.S.; Krotova, L.A.; Ansolis, L.E.; Tzvigun, E.A. In vitro activity of ampicillin, cefoperazone, their combinations with sulbactam and other antimicrobials: Survey of Russian isolates. Int. J. Antimicrob. Agents 1996, 7, 109-117. [CrossRef]

(C) 2019 by the authors. Licensee MDPI, Basel, Switzerland. This article is an open access article distributed under the terms and conditions of the Creative Commons Attribution (CC BY) license (http://creativecommons.org/licenses/by/4.0/). 